\title{
GAME THEORY MODELS FOR SPECTRAL BAND GROUPING AND CLASSIFIER ENSEMBLES FOR HYPERSPECTRAL IMAGE CLASSIFICATION
}

\author{
Lori Mann Bruce, Ph.D. \\ Professor and Dean of the Graduate School, Mississippi State University, U.S.A,
}

\begin{abstract}
This paper investigates the utilization of game theory models for automated analysis of hyperspectral imagery. The author proposes three approaches to using strategic, competitive game theory models for groundcover classification using hyperspectral imagery, including the application of game theory models to (i) hyperspectral band grouping and (ii) pixel classification in a classifier ensemble system. Proposed model (i) uses conflict data filtering based on mutual entropy along with the Nash equilibrium as the means to find a steady state solution. Proposed model (ii) utilizes a strategic coalition game, specifically the weighted majority game (WMG). Both a models are implemented under the assumption that all players are rational. The author incorporates each of the proposed approaches, (i) and (ii), into a multi-classifier decision fusion (MCDF) system for automated ground cover classification with hyperspectral imagery. The paper provides experimental results demonstrating the efficacy of the proposed game theoretic approaches, presenting significant improvements over existing methods.
\end{abstract}

Index Terms - Game theory, hyperspectral, remote sensing, band grouping, feature selection, classification, classifier ensemble, precision agriculture

\section{GAME THEORY FUNDAMENTALS}

In the discipline of information analysis and decision making, game theory is one of the most mature fields, with well-defined and proven mathematical models. As a mathematical tool, game theory has mostly been used to support decision-making in the field of economics. Game theory describes how players accumulate benefits for themselves by employing appropriate strategies in a competitive or cooperative activity where many players participate. The following is an extremely brief introduction to core concepts of game theory, and it serves to define vocabulary and concepts that aid in a discussion of how game theory can be applied to big data analytics for geosciences and remote sensing. Game theory models are abstract descriptions of real-life decision making scenarios. The basic assumptions that underlie these models are that decision makers are rational and strategic, i.e. decision makers pursue well-defined goals and take into account their expectations of other decision makers [4].
In game theory models, the basic entity is a player, which may be interpreted as an individual or as a group of individuals making a decision. Models where primitives represent possible actions of individual players are referred to as noncooperative; models where primitives represent possible joint actions of groups of players are referred to as cooperative. Game theory takes into account the attempts of each player to obtain information about other players' behavior prior to making their own decisions. A strategic game is a model of a scenario where each player chooses their plan of action, and all players' decisions are made simultaneously without knowing the decisions of other players. At the heart of game theory is the assumption of rational behavior. Rational behavior in game theory models is defined as below, when there is no uncertainty [4].

- $A$ is a set of decisions/actions from which a player selects.

- $C$ is a set of possible consequences of the decisions/actions.

- $g: A \rightarrow C$ is a consequence function that associates consequences with decisions/actions.

- $\quad U: C \rightarrow \mathbb{R}$ is a utility function that specifies the player's preferences, defined by a preference relation $\gtrsim$ by the condition $x \gtrsim y$ if and only if $U(x) \geq U(y)$.

Uncertainty can also be introduced into the model by the players being uncertain about optimization parameters, imperfectly informed about events in the game, or uncertain about decisions/actions of other players and/or their consequences. Typically, uncertainty is modeled by the consequence function being a stochastic process that is known to the player and the player makes decisions that maximize the value of the utility function.

Arguably the most commonly used model is the strategic game, which is defined by as below [5].

- $\quad N$ is a set of players.

- $A_{i}$ is a set of decisions/actions available to player $i \in N$.

- For each player $i$, a preference relation $\gtrsim_{i}$ on $A=$ $\times_{j \in N} A_{j}$, the preference relation of player $i$.

And the most famous theory is the Nash equilibrium of a strategic game, which is defined as $\left\langle N,\left(A_{i}\right),\left(\gtrsim_{i}\right)\right\rangle$, a profile $a^{*} \in A$ of decisions/actions with the property that for every player $i \in N$ and $\left(a_{-i}^{*}, a_{i}^{*}\right) \succsim_{i}\left(a_{-i}^{*}, a_{i}\right)$ for all $a_{i} \in A_{i}[4,5]$. That is, a Nash equilibrium is a decision profile $a^{*}$ with the property that no player $i$ can do better choosing a decision/action different from $a_{i}^{*}$, given that every other player $j$ adheres to $a_{j}^{*}$. 


\section{APPLYING GAME THEORY TO FEATURE SELECTION AND CLASSIFICATION}

Game theory, and its mathematical models, can be applied to many areas of decision making in hyperspectral remote sensing. These include campaign planning, routing/mapping sensors, data fusion, feature selection, and classification.

In the past five years, researchers have begun to apply game theory to the problem of multi-source data fusion [6, 7] and feature selection [8]. Feature selection is an especially critical area hyperspectral remote sensing. Many feature selection methods choose top-ranking features while others consider the potential cooperative or conflicting relationships among features. In [8], researchers introduce the use of coalitional game models for feature selection. In [9], Bruce introduces the concept of applying game theory approaches to hyperspectral feature selection.

Classification can also employ game theory, particularly when using classifier ensembles. In this case, a player, $i$, is defined as a single classifier in the ensemble. $A_{i}$, the course of actions, is the classification decision. Consequences to decisions/actions include measures of total classification accuracy and classification confidence. In [10], researchers apply strategic, competitive, game theory models to conduct multi-class classification when utilizing binary, or two-class, support vector machines (SVM). In [11], researchers introduce a new unsupervised classification approach based on cooperative games. Performance metrics included sensitivity, specificity, area under Receiver Operating Characteristics (ROC) curves.

\section{APPLYING GAME THEORY TO HYPERSPECTRAL SIGNATURE CLASSIFICATION}

This paper presents methodologies and experimental results for applying game theory to the different stages of a multi-classifier decision fusion (MCDF) system. The MCDF approach is a "divide and conquer" tactic for classifying a hyperspectral signature, which has been shown to be particularly useful when the amount of labeled ground truth pixels is very small compared to the amount of spectral features available in the hyperspectral image cube [12]. The input hyperspectral signature is partitioned into a set of $N$ band groups. Each band group is then treated independently, being classified with an SVM, resulting in $N$ classification labels. The $N$ labels are then combined into one label using decision fusion. Figure 1 shows a block diagram of the MCDF system. The author investigates the use of game theory models for the (i) spectral band grouping and (ii) classification/decision fusion stages of the MCDF system.

\subsection{Spectral Band Grouping}

Spectral bands within a group and across groups have the potential to be redundant, complementary, or conflicting. When applying strategic, competitive game theory models, a player, $i \in N$, is defined as a band group. The set $A_{i}$, the

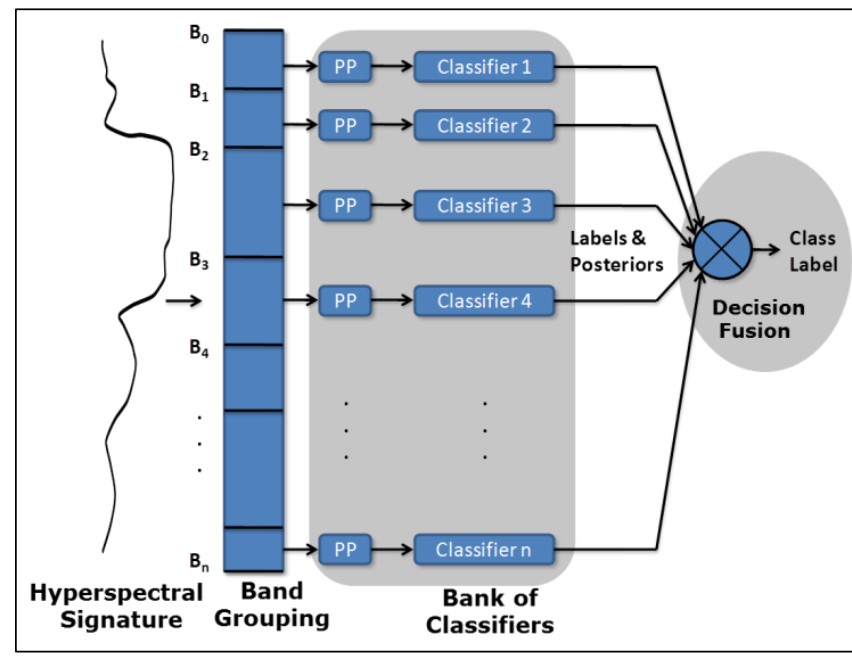

Figure 1. Multi-Classifier Decision Fusion (MCDF) system.

course of actions, is defined as the decision of whether or not to include a spectral band in the group $i$. Consequences to decisions are based on class separability metrics for the group. The proposed system uses conflict data filtering based on mutual entropy and a strategy interaction process of multiple band groups in a conflict environment, the goal of which is to maximize the payoff benefit of multiple groups of the whole system [13]. The "rounds" of the game can be viewed as iterations in an optimization process, which monitors the global performance of the band groups as a whole. In each iteration, each player considers the addition of a band to its group. The mutual entropy is calculated and the bands that provide conflict data are not considered to be reliable and are discarded. Bands that increase a player's ability to maximize the payoff, i.e class separability, are considered for inclusion.

The author uses the Nash equilibrium as the means to find a steady state solution to the band grouping problem, and implements the model under the assumption that all players are rational. As a result, all players, or band groups, operate with the goal of maximizing their own class separability. This personal rationality can violate the class separability, i.e. payoff, of the whole system; thus cooperation mechanisms are introduced to ensure the global payoff is maximized. Thus, the author employs the concept of Pareto efficiency [5]. Pareto-dominant Nash equilibrium indicates collective rationality and the players will converge on the Pareto-dominant Nash equilibrium as the solution of the game.

\subsection{Classification and Decision Fusion}

For the classifier ensemble, a strategic coalition game theoretic model was applied, specifically the weighted majority game (WMG) using the weighted majority rules (WMR), where each classifier participates in a simple coalition game [14]. A player, $i \in N$, is defined as a single classifier in the ensemble. In this study, SVMs were used for 
the classifiers. The set $A_{i}$, the course of actions, is defined as the classification of a spectral band group as class $i$. Consequences to decisions are based on overall classification accuracies, that is the classification accuracy of the MCDF system rather than the singular accuracy of the individual classifier. The "rounds" of the game can be viewed as iterations in an optimization process, which monitors the global performance (classification accuracy) of the MCDF system. Each iteration consists of each player deciding the classification of their spectral bandgroup and those decisions being cast as weighted votes, where weights are calculated using the log-odds solution [14] and the WMR formula is used as the optimal voting aggregation scheme.

\section{EXPERIMENTAL RESULTS}

In this study, three sets of hyperspectral imagery were assessed: (A) the well-known Indian Pines dataset [15], (B) the Washington DC Mall dataset [15], and (C) a dataset of agricultural crops under varying degrees of chemically induced vegetative stress [16].

In each experimental analysis, the classification accuracies of the proposed game theoretic hyperspectral band grouping method, referred to as method (i), is compared to more traditional band grouping methods: uniform partitioning and product of the Bhattacharyya distance and spectral band correlation (BDCORR). For the MCDF, a bank of SVM classifiers utilized along with linear opinion pool (LOP) for the decision fusion. Likewise, for each of the three datasets, the proposed game theoretic classification and decision fusion method, referred to as method (ii), is compared to traditional decision fusion methods: majority vote (MV) and linear opinion pool (LOP). For this portion of the study, the spectral band grouping is carried out using the traditional BDCORR. In all three cases, the proposed game theoretic approaches outperform the two comparison methods.

For dataset (A), the proposed method (i) results in an overall accuracy (OA) of $95 \%$, with uniform partitioning and BDCORR producing an OA of $82 \%$ and $89 \%$, respectively. Likewise for dataset (B), method (i), uniform partitioning, and BDCORR produce an OA of $92 \%, 79 \%$, and $84 \%$, respectively. For dataset $(\mathrm{C})$, which is a very challenging dataset, the proposed method (i), uniform partitioning, and BDCORR produce an OA of $77 \%, 63 \%$, and $58 \%$, respectively. Tables I and II show the confusion matrices for the BDCORR approach and the proposed game theory model approaches, respectively, for dataset (C). Figure 3 shows the classification maps for dataset (C).

For dataset (A), the proposed method (ii) results in an overall accuracy (OA) of $84 \%$, with LOP and MV producing an $\mathrm{OA}$ of $82 \%$ and $71 \%$, respectively. Likewise for dataset (B), method (ii), LOP, and MV produce an OA of $88 \%, 73 \%$, and $84 \%$, respectively. For dataset (C), which is a very challenging dataset, the proposed method (ii), LOP, and MV produce an OA of $61 \%, 60 \%$, and $51 \%$, respectively.

Tables I and II show the confusion matrices for the BDCORR approach and the proposed (i) game theoretic approach to band grouping, respectively, for dataset (C). Tables III and IV show the confusion matrices for the MV approach and the proposed (ii) game theoretic approach to decision fusion, respectively, for dataset (C). Figure 2 shows the classification maps for dataset $(\mathrm{C})$.

\section{CONCLUSIONS}

Game theory, and its mathematical models, can be a very useful tool for automated analysis of hyperspectral imagery. This paper provides an example of utilizing strategic, competitive game theory models for the purpose of spectral band grouping and strategic, coalition game theory models for classifier ensembles using decision fusion. The paper uses each proposed method as a component in a MCDF system for automated ground cover classification with hyperspectral imagery. The experimental results demonstrate that the proposed game theoretic approaches significantly outperform the comparison methods

Table I. Classification confusion matrix for BDCORR method of spectral band grouping in MCDF system for dataset (C).

\begin{tabular}{|l|r|r|r|r|r|r|r|r|}
\hline & $1 \mathrm{x}$ & $.5 \mathrm{x}$ & $.25 \mathrm{x}$ & $.125 \mathrm{x}$ & $.0625 \mathrm{x}$ & $.03125 \mathrm{x}$ & Control & Prod Acc \\
\hline $1 \mathrm{x}$ & 67 & 7 & 3 & 7 & 9 & 5 & 2 & 0.67 \\
\hline $.5 \mathrm{x}$ & 9 & 66 & 16 & 7 & 0 & 1 & 1 & 0.66 \\
\hline $.25 \mathrm{x}$ & 4 & 2 & 66 & 8 & 8 & 6 & 6 & 0.66 \\
\hline $.125 \mathrm{x}$ & 4 & 5 & 7 & 58 & 5 & 13 & 8 & 0.58 \\
\hline $.0625 \mathrm{x}$ & 2 & 0 & 0 & 14 & 58 & 17 & 9 & 0.58 \\
\hline $.03125 \mathrm{x}$ & 1 & 0 & 3 & 8 & 8 & 65 & 15 & 0.65 \\
\hline Control & 4 & 6 & 4 & 9 & 8 & 6 & 63 & 0.63 \\
\hline User Acc & 0.74 & 0.08 & 0.67 & 0.52 & 0.60 & 0.58 & 0.61 & 0.63 \\
\hline
\end{tabular}

Table II. Classification confusion matrix for proposed Game Theory approach (i) of spectral band grouping in MCDF system for dataset (C).

\begin{tabular}{|l|r|r|r|r|r|r|r|r|}
\hline & $1 \mathrm{x}$ & \multicolumn{1}{|c|}{$\mathrm{x}$} & $.25 \mathrm{x}$ & $.125 \mathrm{x}$ & $.0625 \mathrm{x}$ & $.03125 \mathrm{x}$ & Control & Prod Acc \\
\hline $1 \mathrm{x}$ & 88 & 7 & 3 & 2 & 0 & 0 & 0 & 0.88 \\
\hline $.5 \mathrm{x}$ & 5 & 89 & 3 & 1 & 0 & 1 & 1 & 0.89 \\
\hline $.25 \mathrm{x}$ & 1 & 2 & 82 & 9 & 6 & 0 & 0 & 0.82 \\
\hline $.125 \mathrm{x}$ & 1 & 0 & 7 & 83 & 5 & 3 & 1 & 0.83 \\
\hline $.0625 \mathrm{x}$ & 2 & 0 & 0 & 14 & 68 & 7 & 9 & 0.68 \\
\hline $.03125 \mathrm{x}$ & 1 & 0 & 0 & 6 & 7 & 71 & 15 & 0.71 \\
\hline Control & 0 & 0 & 1 & 11 & 12 & 16 & 60 & 0.6 \\
\hline User Acc & 0.90 & 0.07 & 0.85 & 0.66 & 0.69 & 0.72 & 0.70 & 0.77 \\
\hline
\end{tabular}


Table III. Classification confusion matrix for MV method of decision fusion in MCDF system for dataset (C).

\begin{tabular}{|l|r|r|r|r|r|r|r|r|}
\hline & \multicolumn{1}{l|}{ x } & $.5 \mathrm{x}$ & $.25 \mathrm{x}$ & $.125 \mathrm{x}$ & $.0625 \mathrm{x}$ & $.03125 \mathrm{x}$ & Control & Prod Acc \\
\hline $1 \mathrm{x}$ & 56 & 12 & 12 & 9 & 8 & 3 & 0 & 0.56 \\
\hline $.5 \mathrm{x}$ & 11 & 54 & 20 & 7 & 5 & 3 & 0 & 0.54 \\
\hline $.25 \mathrm{x}$ & 4 & 5 & 55 & 10 & 12 & 10 & 4 & 0.55 \\
\hline $.125 \mathrm{x}$ & 4 & 7 & 7 & 52 & 7 & 13 & 10 & 0.52 \\
\hline $.0625 \mathrm{x}$ & 1 & 3 & 0 & 17 & 50 & 22 & 7 & 0.5 \\
\hline $.03125 \mathrm{x}$ & 1 & 3 & 9 & 8 & 12 & 48 & 19 & 0.48 \\
\hline Control & 0 & 3 & 8 & 12 & 15 & 20 & 42 & 0.42 \\
\hline User Acc & 0.73 & 0.62 & 0.50 & 0.45 & 0.46 & 0.40 & 0.51 & 0.51 \\
\hline
\end{tabular}

Table IV. Classification confusion matrix for proposed Game Theory approach (ii) of classification and decision fusion in MCDF system for dataset (C).

\begin{tabular}{|l|r|r|r|r|r|r|r|r|}
\hline & \multicolumn{1}{l|}{$1 \mathrm{x}$} & $.5 \mathrm{x}$ & $.25 \mathrm{x}$ & $.125 \mathrm{x}$ & $.0625 \mathrm{x}$ & $.03125 \mathrm{x}$ & Control & Prod Acc \\
\hline $1 \mathrm{x}$ & 69 & 11 & 8 & 7 & 5 & 0 & 0 & 0.69 \\
\hline $.5 \mathrm{x}$ & 15 & 66 & 13 & 3 & 2 & 1 & 0 & 0.66 \\
\hline $.25 \mathrm{x}$ & 1 & 13 & 63 & 12 & 7 & 3 & 1 & 0.63 \\
\hline $.125 \mathrm{x}$ & 1 & 4 & 13 & 61 & 14 & 6 & 1 & 0.61 \\
\hline $.0625 \mathrm{x}$ & 4 & 0 & 6 & 14 & 61 & 11 & 4 & 0.61 \\
\hline $.03125 \mathrm{x}$ & 1 & 0 & 3 & 8 & 13 & 56 & 19 & 0.56 \\
\hline Control & 0 & 2 & 7 & 11 & 13 & 13 & 54 & 0.54 \\
\hline User Acc & 0.76 & 0.69 & 0.56 & 0.53 & 0.53 & 0.62 & 0.68 & 0.61 \\
\hline
\end{tabular}

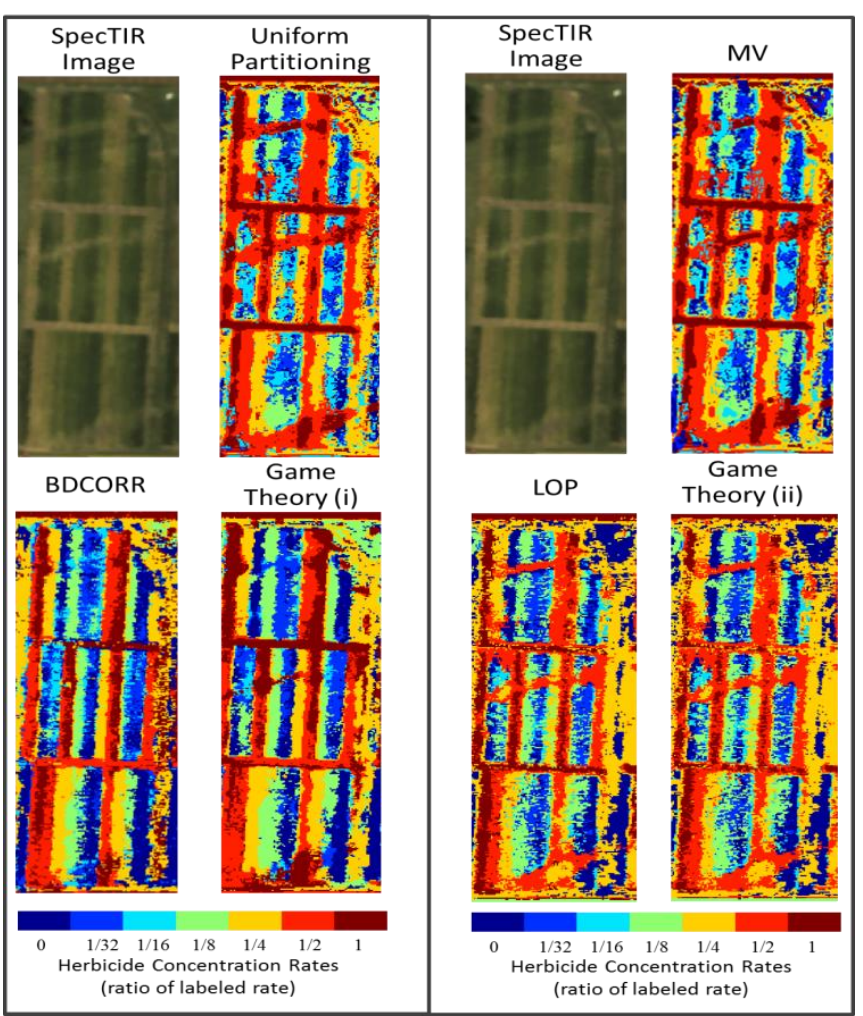

Figure 2. Resulting classification maps for dataset (C) for lefthand-column: Uniform Partitioning, BDCORR, and proposed

Game Theoretic Band Grouping approach (i), and for right-handcolumn: MV, LOP, and proposed Game Theoretic Classification/Decision Fusion approach (ii).

\section{REFERENCES}

[1] R.L. Ackoff, "From data to wisdom," Journal of Applied Systems Analysis, vol. 16, pp. 3-9, 1989.

[2] J. Rowley, "The wisdom hierarchy: representations of the DIKW hierarchy," Journal of Information Science, vol. 33, no. 2, pp. 163-180, 2007.

[3] D. Deptula, Deputy Chief of Staff, Intelligence, Surveillance and Reconnaissance, U.S. Air Force, "RPA in the US Air Force," Air Force Symposium on Academic Opportunities: Developing the Future of UAS/RPA, 2010.

[4] M. J. Osborne, A. Rubinstein, A Course in Game Theory, The MIT Press, Cambridge, Massachusetts, 1994.

[5] M.J. Osborne, An Introduction to Game Theory, Oxford University Press, New York, 2004.

[6] H. Yin, L. Wang, J. Nong, "Survey on Game-theoretic Information Fusion," Proceedings of 7th International Conference on Fuzzy Systems and Knowledge Discovery (FSKD), pp. 21472151, 2010.

[7] N. Xu, X. Wang, "An Information Fusion Method Based on Game Theory," Proceedings of 2nd International Conference on Signal Processing Systems (ICSPS), pp. V1-95-V1-98, 2010.

[8] J. Liu, S.-Y. Lee, "Study on Feature Select Based on Coalitional Game," Proceedings of 2008 International Conference on Neural Networks and Signal Processing, pp. 445-450, 2008.

[9] L. M. Bruce, "Game Theory Applied to Big Data Analytics," Proc. IEEE Geoscience and Remote Sensing Symposium (IGARSS), Melbourne, Australia, July 2013.

[10] M. Petrovskiy, "A Game Theory Approach to Pairwise Classification with Support Vector Machines," Proceedings of 2004 International Conference on Machine Learning and Applications, pp. 115-122, 2004.

[11] A. Torkaman, N. M. Charkari, M. Aghaeipour, "A New Classification Approach Based on Cooperative Game," Proceedings of 14th International CSI Computer Conference, pp. 458-463, 2009.

[12] S. Prasad, L.M. Bruce, "A Divide-and-Conquer Paradigm for Hyperspectral Classification and Target Recognition," Chapter 7 in Optical Remote Sensing - Advances in Signal Processing and Exploitation Techniques, Springer Publishing Company, Berlin, 2011. (ISBN: 978-3-642-14211-6).

[13] N. Xu, X. Wang, "An Information Fusion Method Based on Game Theory," Proceedings of 2nd International Conference on Signal Processing Systems (ICSPS), pp. V1-95-V1-98, 2010.

[14] H.V. Georgiou and M.E. Mavroforakis, "A game-theoretic framework for classifier ensembles using weighted majority voting with local accuracy estimates," https://arxiv.org/abs/1302.0540.

[15] MultiSpec(C) Data Download site:

https://engineering.purdue.edu/ biehl/MultiSpec/hyperspectral.html .

[16] H. Kalluri, S. Prasad, L.M. Bruce "Decision Level Fusion of Spectral Reflectance and Derivative Information for Hyperspectral Classification and Target Recognition," IEEE Transactions on Geoscience and Remote Sensing, vol.48, no.11, pp.4047-4058, November, 2010. 